\title{
Factores de riesgo para la malignidad de los pólipos vesiculares en dos hospitales públicos de Perú
}

\section{Risk factors for gallbladder polyp malignancy in two public hospitals of Peru}

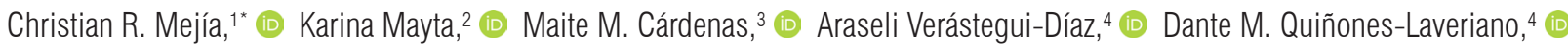 \\ Julio Maravi-Coronado, ${ }^{5}$ (D) Eduardo Monge, ${ }^{5}$ (D) Claudia A. Vera. ${ }^{4}$ (it)
}

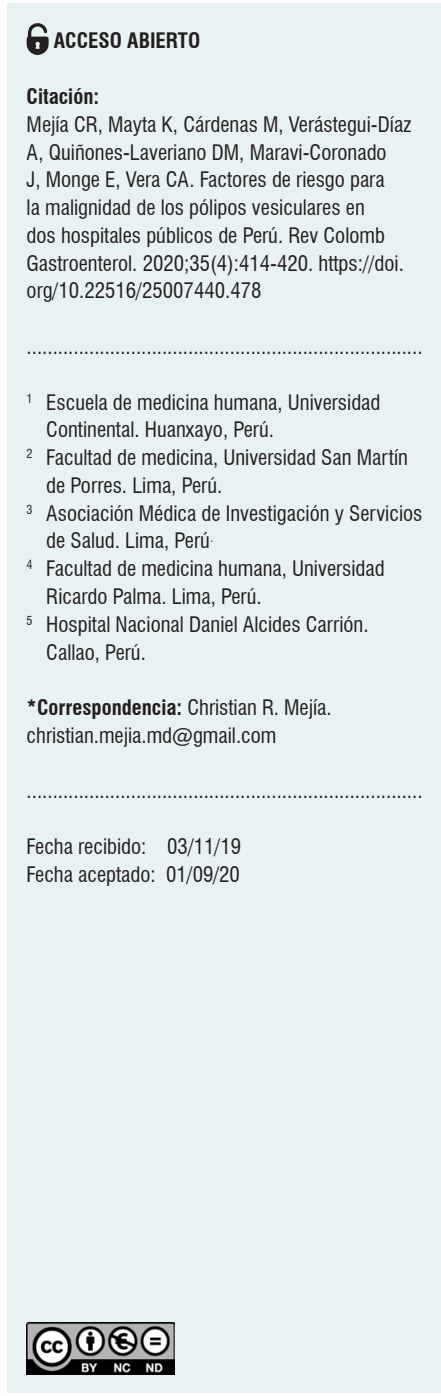

\begin{abstract}
Resumen
Introducción: los pólipos de vesícula biliar, benignos y malignos, en la mayoría de pacientes tienen un diagnóstico generalmente incidental; a través de estudios de imágenes, que no se pueden distinguir con precisión según su grado de malignidad. Objetivo: determinar los factores de riesgo para la malignidad de los pólipos vesiculares en dos hospitales públicos peruanos. Metodología: estudio de cohorte retrospectiva, de datos secundarios, en colecistectomizados del 2004 al 2012 en un hospital de Lima y otro de Callao. Se definió como pólipo maligno según el tipo histopatológico de adenocarcinoma. Se obtuvieron los riesgos relativos y sus intervalos de confianza del $95 \%$ (IC $95 \%$ ). Además, mediante curvas ROC (característica operativa del receptor), se obtuvieron la sensibilidad y especificidad según el tamaño de pólipo. Resultados: de las 368 biopsias, 26 (7\%) fueron adenocarcinomas. La mediana del tamaño de los pólipos fue de $4 \mathrm{~mm}$ (rango: 1-65 $\mathrm{mm}), 176(51 \%)$ tuvieron múltiples pólipos y 85 (23\%) tuvieron litiasis biliar asociada. En el análisis multivariado, se incrementó el riesgo de malignidad por cada milímetro del tamaño del pólipo en $26 \%$ (IC $95 \%$ : $14 \%-40 \%$, valor $p<0,001$ ) y del tamaño de la pared vesicular en $182 \%$ (IC 95\%:46\%-445\%, valor $p$ : $0,002)$, ajustados por la edad del paciente, la litiasis y el tamaño vesicular. Para un tamaño de $6 \mathrm{~mm}$ se tuvo una sensibilidad de $81 \%$ y especificidad del $85 \%$. Conclusión: se concluye que el tamaño del pólipo y el grosor de la pared vesicular estuvieron asociados con la malignidad de pólipos vesiculares.
\end{abstract}

\section{Palabras clave}

Neoplasias de la vesícula biliar, pólipos, adenocarcinoma, factores de riesgo (Fuente: DeCS BIREME).

\begin{abstract}
Introduction: In most patients, gallbladder polyps, both benign and malignant, are usually an incidental finding. However, imaging studies cannot accurately establish their degree of malignancy. Objective: To determine the risk factors for gallbladder polyp malignancy in two Peruvian public hospitals. Methodology: Retrospective cohort study conducted on secondary data from patients who underwent cholecystectomy between 2004 and 2012 in Lima and another in Callao, Peru. The malignancy of the polyp was established according to the histopathological type of adenocarcinoma. Relative risks and their $95 \%$ confidence intervals $(95 \% \mathrm{Cl})$ were obtained. Moreover, ROC curves were used to determine sensitivity and specificity according to the size of the polyp. Results: Of 368 biopsies, $26(7 \%)$ were adenocarcinomas. The median size of the polyps was $4 \mathrm{~mm}$ (range: $1-65 \mathrm{~mm}) .176$ patients $(51 \%)$ had multiple polyps, and $85(23 \%)$ had associated gallstones. Multivariate analysis showed that the risk of malignancy increased by $26 \%(95 \% \mathrm{Cl}: 14-40 \%$, pvalue:<0.001) per millimeter of polyp size and by $182 \%(95 \% \mathrm{Cl}: 46-445 \%$, $\mathrm{p}$-value $=0.002)$ based on vesicular wall size, adjusted for patient age, lithiasis and vesicular size. For a size of $6 \mathrm{~mm}$, sensitivity was $81 \%$, and specificity was $85 \%$. Conclusion: The size of the polyp and the thickness of the vesicular wall are associated with the malignancy of vesicular polyps.
\end{abstract}

\section{Keywords}

Gallbladder neoplasms, polyps, adenocarcinoma, risk factors. 


\section{INTRODUCCIÓN}

El cáncer de vesícula biliar se encuentra en el quinto lugar dentro de los cánceres del tracto digestivo más frecuentes a nivel mundial (1), teniendo un mal pronóstico una vez que se detecta, siendo el tiempo de supervivencia promedio de 6 meses $(2,3)$. Su incidencia es variable, desde las más bajas en países desarrollados hasta los reportes de altas incidencias en ciertas etnias sudamericanas (4). En el Perú se encuentra entre las neoplasias más frecuente en mujeres de la tercera edad (5).

Uno de los factores más asociados es la presencia de pólipos vesiculares, debido a que del $4 \%$ al $7 \%$ de individuos sanos presentan pólipos en la vesícula biliar (6). De los que serán catalogados como malignos, el $85 \%$ son de tipo adenocarcinoma $(7,8)$ y generalmente son diagnosticados de forma incidental a través del ultrasonido abdominal (9). Otros factores asociados con la malignidad son la edad mayor a 60 años y el tamaño mayor a $10 \mathrm{~mm}(10-12)$, la presencia de síntomas $(11,13)$, la litiasis vesicular $(10,14)$, la cantidad de pólipos $(11,14)$, entre otros (15).

En nuestro medio los estudios del tema describen las características, sin realizar análisis para encontrar la asociación entre sus variables y dicha patología, como el que se realizó en una población que acude a un establecimiento privado de salud en una zona residencial (16). Por todo lo mencionado, el objetivo del estudio fue determinar los factores de riesgo para la malignidad de los pólipos vesiculares en dos hospitales públicos peruanos.

\section{MATERIALES Y MÉTODOS}

\section{Diseño y población}

Se desarrolló un estudio observacional tipo cohorte retrospectiva, usando un análisis de datos secundarios. Las bases de datos se obtuvieron de los archivos de los hospitales Nacional Arzobispo Loayza (HNAL) y Nacional Daniel Alcides Carrión (HNDAC), durante el período 2004-2012; estos hospitales son públicos y a ellos acuden en su mayoría personas de los sectores socioeconómicos C, D y E.

Se incluyeron los reportes de las biopsias vesiculares de los pacientes que fueron colecistectomizados y que tuvieron su posterior diagnóstico anatomopatológico confirmado de pólipos en la vesícula biliar. Se excluyó a los que no tuvieron los datos principales completos. Se usó un muestreo no probabilístico de tipo censal.

\section{Procedimientos y variables}

Se solicitó el permiso en ambas sedes para el uso de los datos, se generó una base en el programa Microsoft Excel para
Windows 2013, posterior a ello se realizó la limpieza de los mismos, seleccionando aquellos que cumplían los criterios de selección. La investigación fue aprobada por un comité de local avalado por el instituto nacional de salud peruano.

La variable dependiente fue el diagnóstico de malignidad de los pólipos según el tipo anatomopatológico de los pólipos de la vesícula biliar. Se consideraron benignos los pólipos de tipo adenoma, hiperplásico, colesterolósico e inflamatorio; y como malignos los de tipo adenocarcinoma.

Las variables independientes del estudio fueron la edad y el sexo del paciente (para el análisis estadístico, el sexo femenino fue considerado de interés); las características anatomopatológicas: tamaño del pólipo (medido en milímetros, en los casos de pólipos múltiples se tomó como medida al mayor de los tamaños), la cantidad de pólipos (considerándose como múltiple la presencia de 2 o más pólipos), además de las características vesiculares: tamaño del diámetro vesicular (medida en centímetros según el largo de la vesícula) y el grosor de la pared vesicular (medida en milímetros según el grosor de la pared en el lugar del pólipo).

\section{Análisis estadístico}

Se usó el programa Stata 11,1 para Windows para los análisis. Las variables categóricas fueron descritas mediante las frecuencias y porcentajes; y las variables cuantitativas, mediante la media y desviación estándar; para las diferencias de las variables categóricas y cuantitativas se obtuvieron los valores $p$ mediante las pruebas estadísticas el chi cuadrado $\left(\chi^{2}\right)$ y t de Student. Para el análisis bi- y multivariado se obtuvieron los riesgos relativos crudos (RRc) y ajustados (RRa) con sus intervalos de confianza del $95 \%$ (IC $95 \%$ ). De manera exploratoria se utilizó la curva de característica operativa del receptor (ROC), para poder hallar la sensibilidad y especificidad según los puntos de corte del tamaño de los pólipos vesiculares. Se consideró a los valores $p<0,05$ como estadísticamente significativos.

\section{RESULTADOS}

De las 368 biopsias de pacientes colecistectomizados con diagnóstico de pólipos vesiculares, 264 (71,7\%) fueron del HNAL y 104 (28,3\%) del HNDAC; 288 (78,3\%) fueron mujeres y la edad promedio fue de 45,5 años $( \pm 15,5$ años, rango: 12-86 años). La mediana del tamaño de los pólipos fue de $4 \mathrm{~mm}$ (rango: 1-65 mm), $176(50,7 \%)$ tuvieron múltiples pólipos y $85(23,1 \%)$ tuvieron litiasis biliar asociada. $26(7,1 \%)$ fueron pólipos malignos del tipo adenocarcinoma, los otros fueron de tipo benigno: 237 (64,6\%) de tipo colesterolósico, $53(14,4 \%)$ de tipo hiperplásico, 48 $(13,1 \%)$ de tipo adenoma y $3(0,8 \%)$ de tipo inflamatorio. 
Según la malignidad, hubo diferencias según la edad del paciente $(p=0,001)$, el tamaño del pólipo $(p<0,001)$, la litiasis asociada $(p<0,001)$, el tamaño de la $(p=0,001)$ y el grosor de la pared de la vesícula biliar $(p<0,001)$ (Tabla 1$)$.

Analizando según el tipo de pólipo vesicular, la edad $(p=$ $0,006)$, el tamaño de los pólipos $(p<0,001)$, el grosor de la pared vesicular $(p<0,001)$ y el tamaño de la vesícula $(p<$ $0,001)$ tuvieron diferencias estadísticamente significativas en sus medianas (Figura 1).

Valores $p$ obtenidos con la prueba estadística ANOVA para determinar la diferencia en los promedios de cada variable:

- 1A: según la edad del paciente (años cumplidos);

- 1B: según el tamaño del pólipo (mm);

- 1C: según el tamaño de la vesícula biliar $(\mathrm{cm})$;

- 1D: según el grosor de la pared de la vesícula biliar (mm).

En el análisis bivariado, el tamaño del pólipo $(p<0,001)$, el grosor de la pared vesicular $(p<0,001)$, la litiasis vesicular $(p<0,001)$, la edad del paciente $(p=0,003)$ y el tamaño de la vesícula $(p=0,004)$ estuvieron asociados con la malignidad del pólipo vesicular. En el análisis multivariado, se incrementó el riesgo de malignidad por cada milímetro del
Tabla 1. Variables según el tipo de pólipo encontrado en pacientes colecistectomizados en dos hospitales peruanos

\begin{tabular}{|c|c|c|c|}
\hline \multirow[t]{2}{*}{ Variable } & \multicolumn{2}{|c|}{ Tipo de pólipo } & \multirow[t]{2}{*}{ Valor $p$} \\
\hline & $\begin{array}{c}\text { Maligno* } \\
\text { n (\%) }\end{array}$ & $\begin{array}{c}\text { Benigno } \\
n(\%)\end{array}$ & \\
\hline $\begin{array}{l}\text { Sexo } \\
\text { - Femenino } \\
\text { - Masculino }\end{array}$ & $\begin{array}{l}63(85,1) \\
11(14,9)\end{array}$ & $\begin{array}{c}225(76,8) \\
68(23,2)\end{array}$ & $0,119^{* * *}$ \\
\hline Edad (años) ${ }^{* *}$ & $51,6 \pm 17,7$ & $43,8 \pm 14,5$ & $0,001^{* * * *}$ \\
\hline Tamaño de pólipos (mm)* & $10,7 \pm 11,0$ & $4,0 \pm 3,0$ & $<0,001^{* * * *}$ \\
\hline $\begin{array}{l}\text { Cantidad de pólipos } \\
\text { - Múltiple } \\
\text { - Único }\end{array}$ & $\begin{array}{l}25(36,8) \\
43(63,2)\end{array}$ & $\begin{array}{l}151(53,9) \\
129(46,1)\end{array}$ & $0,011^{* * *}$ \\
\hline $\begin{array}{l}\text { Litiasis asociada } \\
\text { - Sí } \\
\text { - No }\end{array}$ & $\begin{array}{l}35(47,3) \\
39(52,7)\end{array}$ & $\begin{array}{c}50(17,1) \\
243(82,9)\end{array}$ & $<0,001^{* \star *}$ \\
\hline Tamaño vesicular $(\mathrm{cm})^{\star *}$ & $7,9 \pm 1,9$ & $7,0 \pm 1,7$ & $<0,001^{* * * *}$ \\
\hline $\begin{array}{l}\text { Grosor de pared vesicular } \\
(\mathrm{mm})^{* *}\end{array}$ & $3,5 \pm 2,3$ & $2,4 \pm 0,8$ & $<0,001^{* * * *}$ \\
\hline
\end{tabular}

${ }^{*}$ Pólipo maligno: tipo adenocarcinoma + adenoma. ${ }^{* *}$ Mediana y rango. *** Obtenido con prueba $\chi^{2} .{ }^{* * * *}$ Obtenido mediante prueba t de Student.
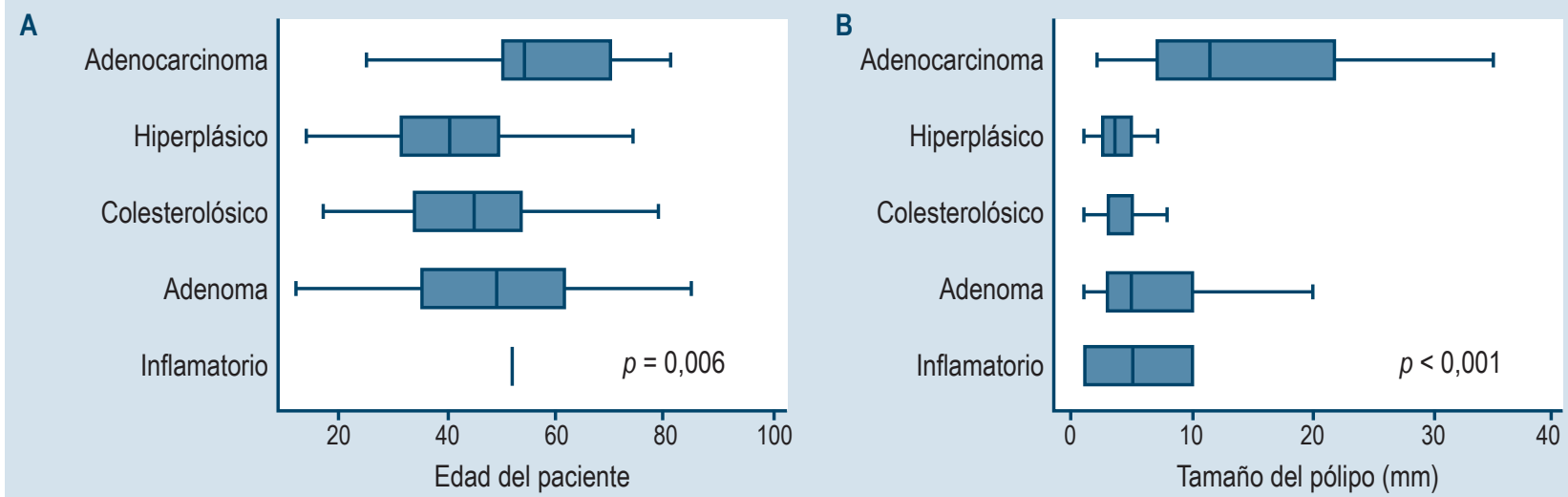

C

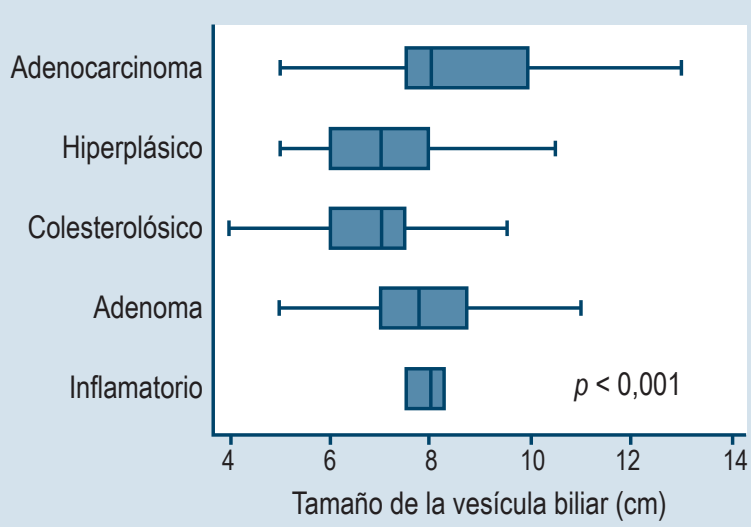

D

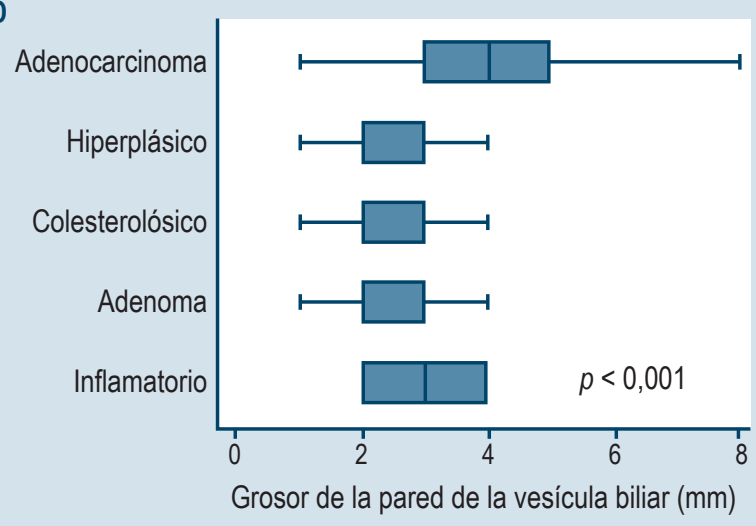

Figura 1. Diferencias de valores según el tipo de pólipo vesicular en pacientes colecistectomizados en dos hospitales de Lima y Callao. 
tamaño del pólipo en $26 \%$ (IC $95 \%$ : $14 \%-40 \%$, valor $p<$ 0,001 ) y del tamaño de la pared vesicular en $182 \%$ (IC $95 \%$ : $46 \%-445 \%$, valor $p=0,002)$, ajustados por la edad del paciente, la litiasis y el tamaño vesicular (Tabla 2).

Tabla 2. Factores de riesgo para la malignidad de pólipos vesiculares en pacientes colecistectomizados en dos hospitales de Lima y Callao

\begin{tabular}{|c|c|c|c|c|}
\hline \multirow[t]{2}{*}{ Variable } & \multicolumn{2}{|c|}{ Análisis bivariado } & \multicolumn{2}{|c|}{ Análisis multivariado } \\
\hline & Valor $p$ & $\operatorname{RRc}($ IC $95 \%)$ & Valor $p$ & RRa (IC $95 \%)$ \\
\hline $\begin{array}{l}\text { Tamaño del } \\
\text { pólipo* (mm) }\end{array}$ & $<0,001$ & $1,27(1,18-1,37)$ & $<0,001$ & $1,23(1,12-1,34)$ \\
\hline $\begin{array}{l}\text { Grosor de } \\
\text { pared* }^{*}(\mathrm{~mm})\end{array}$ & $<0,001$ & $1,98(1,51-2,59)$ & 0,009 & $1,74(1,15-2,62)$ \\
\hline Pólipo múltiple & 0,012 & $0,50(0,29-0,86)$ & 0,022 & $0,37(0,16-0,87)$ \\
\hline $\begin{array}{l}\text { Tamaño } \\
\text { vesicular* }(\mathrm{cm})\end{array}$ & 0,001 & $1,31(1,12-1,53)$ & 0,032 & $1,36(1,03-1,81)$ \\
\hline Litiasis vesicular & $<0,001$ & $4,36(2,52-7,55)$ & 0,435 & $1,42(0,59-3,41)$ \\
\hline Edad* (años) & 0,001 & $1,03(1,01-1,06)$ & 0,316 & $1,01(0,99-1,04)$ \\
\hline Sexo femenino & 0,122 & $1,73(0,86-3,47)$ & 0,577 & $1,36(0,46-4,05)$ \\
\hline
\end{tabular}

${ }^{*}$ Media y desviación estándar/valor $p$. IC $95 \%$ : intervalo de confianza del $95 \%$; RRa: riesgo relativo ajustado; RRc: riesgo relativo crudo.

La Figura 2 muestra las curvas ROC para el tamaño del pólipo $(A=0,78)$, grosor de la pared $(B=0,67)$, tamaño de la vesícula $(C=0,66)$ y con todas las variables en el modelo multivariado $(\mathrm{D}=0,86)$.

Mediante la curva ROC se encontraron los puntos de corte para la variable continua de tamaño del pólipo; el mejor punto de corte se daría a un tamaño de $6 \mathrm{~mm}$, con una sensibilidad de $80,8 \%$ y especificidad de $84,9 \%$ (Tabla 3 ).

Tabla 3. Sensibilidad y especificidad según el tamaño de los pólipos malignos

\begin{tabular}{|cccccc|}
$\begin{array}{c}\text { Tamaño } \\
\text { del pólipo } \\
\text { (mm) }\end{array}$ & Sensibilidad Especificidad & VPP & VPN & $\begin{array}{c}\text { Área } \\
\text { ROC }\end{array}$ \\
\hline$\geq 3$ & $0 \%$ & $100 \%$ & $0 \%$ & $80,1 \%$ & $57,4 \%$ \\
$\geq 4$ & $0 \%$ & $100 \%$ & $0 \%$ & $80,1 \%$ & $66,8 \%$ \\
$\geq 5$ & $0 \%$ & $100 \%$ & $0 \%$ & $80,1 \%$ & $72,6 \%$ \\
\hline$\geq 6$ & $56,3 \%$ & $89,1 \%$ & $56,3 \%$ & $89,1 \%$ & $72,7 \%$ \\
$\geq 7$ & $53,5 \%$ & $91,2 \%$ & $60,3 \%$ & $88,7 \%$ & $72,4 \%$ \\
$\geq 8$ & $50,7 \%$ & $93,0 \%$ & $64,3 \%$ & $88,3 \%$ & $71,8 \%$ \\
$\geq 9$ & $45,1 \%$ & $94,7 \%$ & $68,1 \%$ & $87,4 \%$ & $69,9 \%$ \\
$\geq 10$ & $45,1 \%$ & $95,4 \%$ & $71,1 \%$ & $87,5 \%$ & $70,3 \%$
\end{tabular}

VPN: valor predictivo negativo; VPP: valor predictivo positivo.

\section{DISCUSIÓN}

El $7 \%$ de los pólipos vesiculares fue de tipo maligno y esto es importante debido a que en la mayoría de los pacientes el diagnóstico de pólipos vesiculares es incidental, generalmente luego de un ultrasonido abdominal de rutina o después de haberse realizado una colecistectomía por litiasis biliar o por cólico biliar (17). Sin embargo, debido al desarrollo nuevos equipos de diagnóstico por imágenes, la detección de pólipos vesiculares es cada vez más común (11). Aunque la mayoría de los pólipos de vesícula biliar son benignos, una de las principales preocupaciones es la diferenciación de las masas malignas, debido a que el carcinoma de vesícula biliar generalmente tiene una presentación tardía y pronóstico sombrío, es por ello que deben detectarse tempranamente para cambiar el pronóstico (18).

El tamaño del pólipo estuvo asociado con su malignidad, siendo esto similar a la mayoría de resultados propuestos por estudios similares $(11,19)$; existiendo casi $13 \mathrm{~mm}$ de diferencia entre los promedios según la malignidad. Estudios reportan que el tamaño del pólipo $\geq 10 \mathrm{~mm}$ es un factor predictor de malignidad (20) y hay estudios que señalan que en sus poblaciones de pólipos malignos hallaron entre el $45 \%$ y $67 \%$ de casos un tamaño mayor de $10-15 \mathrm{~mm}(10,21-23)$; incluso, en algunas investigaciones se realizó un seguimiento temporal a los pacientes con pólipos menores de $10 \mathrm{~mm}$ y se concluyó que no se reportaron adenocarcinomas o progresión a malignidad después de este tiempo de seguimiento (24). No obstante, en nuestro estudio se encontraron varios reportes de malignidad en pólipos menores de $5 \mathrm{~mm}$, por lo que se realizó de manera exploratoria los puntos de corte para cada medida mediante la curva ROC y se observó que la mejor medida se halló en pólipos con $\geq 6 \mathrm{~mm}$, teniendo una sensibilidad y especificidad aceptables. Esto coincide con el estudio de Zielinski y colaboradores en una población estadounidense (25), en el que recomienda tomar en consideración la resección quirúrgica de pólipos de vesícula biliar $\geq 6 \mathrm{~mm}$, basado en pruebas preoperatorias de ultrasonido. Además, las evidencias de malignidad en pólipos $>6 \mathrm{~mm}$ son fácilmente observables durante las biopsias o exploraciones realizadas por gastroenterólogos entrenados, pero no lo son tanto en estudios radiológicos (26).

El grosor de la pared vesicular fue otro factor asociado con la malignidad, esto concuerda con los hallazgos de Kim y colaboradores (27), que concluyeron que la pared con grosor $>10 \mathrm{~mm}$ es un factor predictivo para engrosamiento neoplásico de la pared vesicular; del mismo modo, Aldouri y colaboradores (28) señalaron que el grave engrosamiento de la pared vesicular se asocia con una probabilidad más alta de desarrollar cáncer de vesícula biliar. Sun y colaboradores (29) mencionaron entre las indicaciones quirúrgicas 

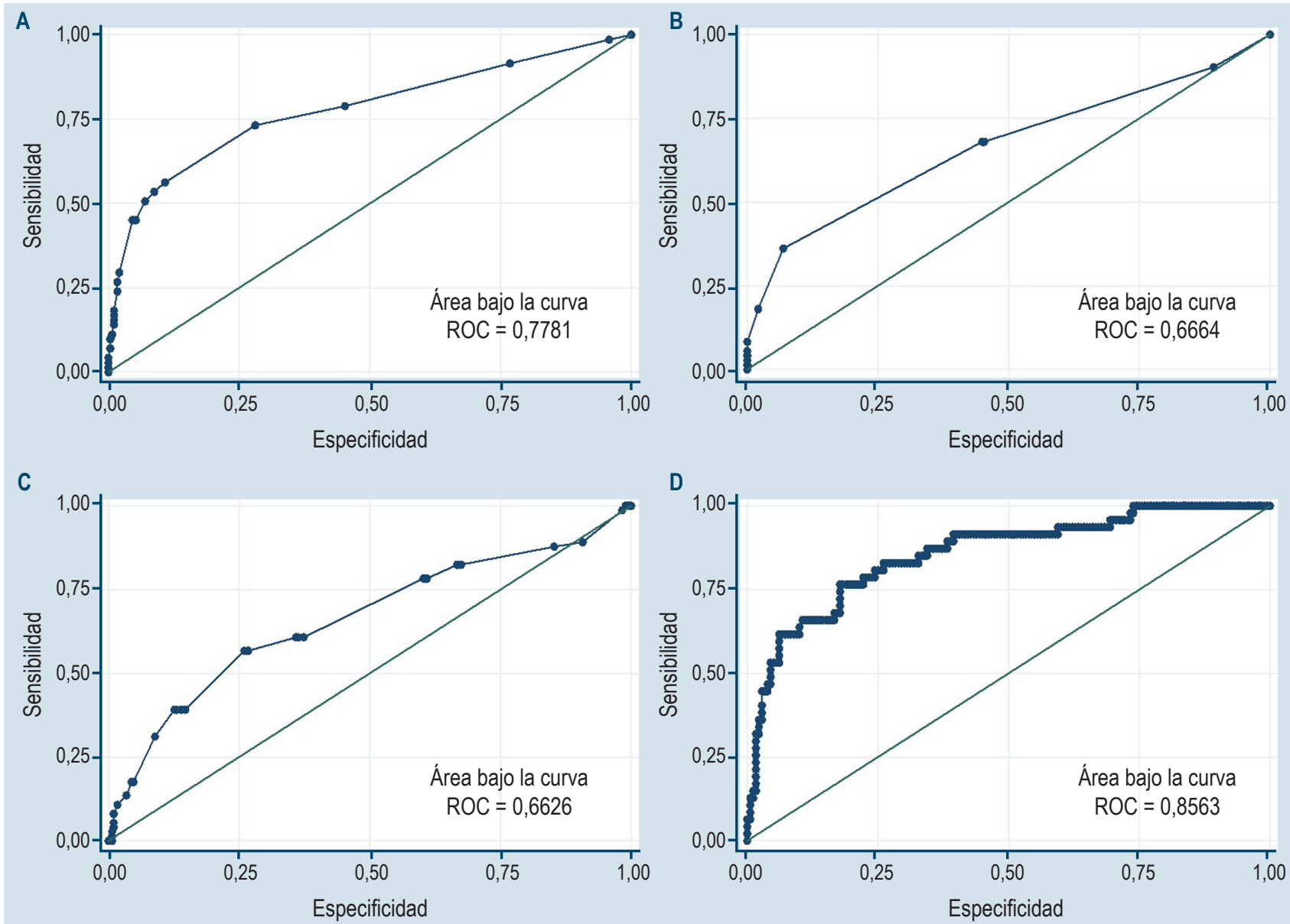

Figura 2. Curvas ROC según el tamaño de pólipo $(A=0,78)$, grosor de la pared $(B=0,67)$, tamaño de la vesícula $(C=0,66)$ y con todas las variables en el modelo multivariado $(\mathrm{D}=0,86)$.

de pólipos vesiculares a aquellos que presenten una pared vesicular engrosada localmente en forma irregular. Por todo lo anterior, se sugiere el estudio del grosor de la pared vesicular en posteriores investigaciones, con el fin de poder determinar su importancia con respecto a la presencia de pólipos malignos.

La diferencia de sexo en nuestro estudio no tuvo significancia como factor de riesgo de malignidad; sin embargo, se pudo observar una predominancia femenina en el grupo de pólipos malignos, lo cual es acorde a los hallazgos de Manrique y colaboradores (30) en su estudio de cáncer de vesícula biliar en una población arequipeña. Esto puede deberse a los diferentes reportes en torno a esta variable, algunos denotan la predominancia masculina sobre la femenina (31-34) y otros de forma inversa $(11,14,35)$. La edad tampoco resultó asociada, a pesar de que algunos estudios sí lo demuestran $(10,11,28)$; ni tampoco la pre- sencia de litiasis vesicular, que no siempre está asociada con la malignidad (11).

Una de las limitaciones encontradas en el estudio fue el sesgo de operador-dependiente, ya que las muestras las evaluaron distintos médicos antes del registro en la base de datos; no obstante, como los médicos patólogos se encuentran altamente capacitados y la patología a evaluar no es causa de alta duda diagnóstica, se consideró que esto no repercutió en gran medida en los resultados obtenidos.

Con base en los datos analizados, se concluye que el tamaño del pólipo y el grosor de la pared vesicular estuvieron asociados con la malignidad de los pólipos vesiculares.

\section{Agradecimientos}

Agradecemos a Emperatriz Centeno por colaborar en la elaboración de algunas secciones del artículo. 


\section{REFERENCIAS}

1. Peña Dávila FE, Sánchez Renteria FA, Fernandez Mogollon J, Rodríguez Rodríguez MR. Frecuencia y perfil clínico de cáncer de vesícula biliar en pacientes colecistectomizados en 3 hospitales referenciales de Chiclayo entre 2011 y 2015. Rev gastroenterol Perú. 2017;37(2):142-5.

2. Navarro Rosenblatt D, Durán Agüero S. Cáncer de vesícula biliar en Chile y factores nutricionales de riesgo. Nutr Hosp. 2016;33(1):105-110.

https://doi.org/10.20960/nh.37

3. Uribe M, Heine TC, Brito MF, Bravo LD. Actualización en cáncer de vesícula biliar. Revista Médica Clínica Las Condes. 2013;24(4):638-643. https://doi.org/10.1016/S0716-8640(13)70202-5

4. Torres Rodríguez JK. Características ecográficas y anatomopatológicas en pacientes colecistectomizados por pólipo vesicular en el Hospital Nacional Edgardo Rebagliati Martins de enero 2016 a julio del 2018. Lima: Universidad Peruana Unión; 2019.

5. Datos Epidemiológicos Perú cáncer 2000-2009 [Internet]. INEN [acceso el 20 de noviembre de 2016]. Disponible en: http://www.inen.sld.pe/portal/estadisticas/datosepidemiologicos.html

6. Contreras Castro E, Alfaro Fernández P, Contreras Castro F, Luna Victoria R, Contreras Alomía I. Correlación entre diagnóstico ecográfico e histopatológico de poliposis vesicular en la Clínica Good Hope 2008-2014. Horiz Med. 2016;16(2):27-32. https://doi.org/10.24265/horizmed.2016.v16n2.05

7. Ramírez AS, Martínez E, Román R, Gamarra E, Villalba W. Cáncer de la vesícula biliar. Experiencia de 10 años del Instituto Nacional del Cáncer. Rev. Cir. Parag. 2016;40(2):8-11. http://dx.doi.org/10.18004/sopaci.noviembre.8-11

8. Justo I, Marcacuzco A, Nutu OA, Manrique A, Calvo J, Caso Ó, Cambra F, García A, Jiménez C. Análisis retrospectivo en pacientes con cáncer de vesícula biliar: tratamiento quirúrgico y supervivencia en función del estadio tumoral. Rev Esp Enferm Dig. 2018;110(8):485-492. https://doi.org/10.17235/reed.2018.5435/2017

9. Pina L, Lagos H, Quiche G, Alle L, Sarotto LE. Carcinoma incidental de vesícula biliar en un hospital universitario. Acta Gastroenterol Latinoam. 2017;47(3):190-193.

10. Wu T, Sun Z, Jiang Y, Yu J, Chang C, Dong X, Yan S. Strategy for discriminating cholesterol and premalignancy in polypoid lesions of the gallbladder: a single-centre, retrospective cohort study. ANZ J Surg. 2019;89(4):388-392. https://doi.org/10.1111/ans.14961

11. Sarici IS, Duzgun O. Gallbladder polypoid lesions $>15 \mathrm{~mm}$ as indicators of $\mathrm{T} 1 \mathrm{~b}$ gallbladder cancer risk. Arab J Gastroenterol. 2017;18(3):156-158. https://doi.org/10.1016/j.ajg.2017.09.003

12. Shin SR, Lee JK, Lee KH, Lee KT, Rhee JC, Jang K-T, Kim SH, Choi DW. Can the growth rate of a gallbladder polyp predict a neoplastic polyp? J Clin Gastroenterol. 2009; 43(9):865-8. https://doi.org/10.1097/MCG.0b013e31819359aa

13. Lee KF, Wong J, Li JCM, Lai PBS. Polypoid lesions of the gallbladder. Am J Surg. 2004;188(2):186-90. https://doi.org/10.1016/j.amjsurg.2003.11.043

14. Shah J. Postoperative Histopathology Findings of Ultrasonographically diagnosed Gallbladder Polyp In 32 Patients. The Internet Journal of Third World Medicine. 2010;9(1):1-5. https://doi.org/10.5580/5be

15. Lai HC, Chang SN, Lin CC, Chen CC, Chou JW, Peng CY, Lai SW, Sung FC, Li YF. Does diabetes mellitus with or without gallstones increase the risk of gallbladder cancer? Results from a population-based cohort study. J Gastroenterol. 2013; 47(8); 856-65. https://doi.org/10.1007/s00535-012-0683-z

16. Tannous MB, Arróspide MT, Huerta-Mercado TJ, Levy YS. Gallbladder polyps: Clinical and pathological features in Cholecystectomy patients in the Anglo American clinic in the period of 1999-2007. Rev Gastroenterol Peru. 2011;31(1):32-7.

17. Gurusamy KS, Abu-Amara M, Farouk M, Davidson BR. Cholecystectomy for gallbladder polyp. Cochrane Database Syst Rev. 2009; (1):CD007052. https://doi.org/10.1002/14651858.CD007052.pub2

18. Myers RP, Shaffer EA, Beck PL. Gallbladder polyps: epidemiology, natural history and management. Can J Gastroenterol. 2002;16(3):187-94. https://doi.org/10.1155/2002/787598

19. Matos ASB de, Baptista HN, Pinheiro C, Martinho F. Gallbladder polyps: how should they be treated and when?. Rev Assoc Med Bras. 2010;56(3):318-21. https://doi.org/10.1590/S0104-42302010000300017

20. Sarkut P, Kilicturgay S, Ozer A, Ozturk E, Yilmazlar T. Gallbladder polyps: factors affecting surgical decision. World J Gastroenterol. 2013;19(28):4526-30. https://doi.org/10.3748/wjg.v19.i28.4526

21. Chattopadhyay D, Lochan R, Balupuri S, Gopinath B-R, Wynne K-S. Outcome of gall bladder polypoidal lesions detected by transabdominal ultrasound scanning: a nine year experience. World J Gastroenterol. 2005;11(14):2171-3. https://doi.org/10.3748/wjg.v11.i14.2171

22. Yeh CN, Jan YY, Chao TC, Chen MF. Laparoscopic cholecystectomy for polypoid lesions of the gallbladder: a clinicopathologic study. Surgical Laparoscopy Endoscopy \& Percutaneous Techniques. 2001;11(3):176-81. https://doi.org/ 10.1097/00019509-200106000-00005

23. Mainprize KS, Gould SW, Gilbert JM. Surgical management of polypoid lesions of the gallbladder. British Journal of Surgery. 2000;87(4):414-7. https://doi.org/10.1046/j.1365-2168.2000.01363.x 
24. Csendes A, Burgos AM, Csendes P, Smok G, Rojas J. Late Follow-Up of Polypoid Lesions of the Gallbladder Smaller Than 10 mm. Ann Surg. 2001;234(5):657-60. https://doi.org/10.1097/00000658-200111000-00011

25. Zielinski MD, Atwell TD, Davis PW, Kendrick ML, Que FG. Comparison of surgically resected polypoid lesions of the gallbladder to their pre-operative ultrasound characteristics. J Gastrointest Surg. 2009;13(1):19-25. https://doi.org/10.1007/s11605-008-0725-2

26. Nagata K, Endo S, Honda T, Yasuda T, Hirayama M, Takahashi S, Kato T, Horita S, Furuya K, Kasai K, Matsumoto H, Kimura Y, Utano K, Sugimoto H, Kato H, Yamada R, Yamamichi J, Shimamoto T, Ryu Y, Matsui O, Kondo H, Doi A, Abe T, Yamano HO, Takeuchi K, Hanai H, Saida Y, Fukuda K, Näppi J, Yoshida H. Accuracy of CT Colonography for Detection of Polypoid and Nonpolypoid Neoplasia by Gastroenterologists and Radiologists: A Nationwide Multicenter Study in Japan. Am J Gastroenterol. 2017;112(1):163-171. https://doi.org/10.1038/ajg.2016.478

27. Kim HJ, Park JH, Park DI, Cho YK, Sohn CI, Jeon WK, Kim BI, Choi SH. Clinical usefulness of endoscopic ultrasonography in the differential diagnosis of gallbladder wall thickening. Dig Dis Sci. 2012;57(2):508-15. https://doi.org/10.1007/s10620-011-1870-0

28. Aldouri AQ, Malik HZ, Waytt J, Khan S, Ranganathan K, Kummaraganti S, Hamilton W, Dexter S, Menon K, Lodge JP, Prasad KR, Toogood GJ. The risk of gallbladder cancer from polyps in a large multiethnic series. Eur J Surg Oncol. 2009;35(1):48-51.

https://doi.org/10.1016/j.ejso.2008.01.036
29. Sun X-J, Shi J-S, Han Y, Wang J-S, Ren H. Diagnosis and treatment of polypoid lesions of the gallbladder: report of 194 cases. HBPD INT. 2004;3(4):591-4.

30. Manrique RRG, Camapaza YIC, Torres FS, Apaza YMO. Cáncer de vesícula biliar según tipo histológico y clasificación TNM en Arequipa, Perú. Acta Médica Peruana. 2012;29(1):23-7.

31. Segawa K, Arisawa T, Niwa Y, Suzuki T, Tsukamoto Y, Goto H, Hamajima E, Shimodaira M, Ohmiya N. Prevalence of gallbladder polyps among apparently healthy Japanese: ultrasonographic study. Am J Gastroenterol. 1992;87(5):630-3.

32. Yang H-L, Kong L, Hou L-L, Shen H-F, Wang Y, Gu X-G, Qin JM, Yin PH, Li Q. Analysis of risk factors for polypoid lesions of gallbladder among health examinees. World J Gastroenterol. 2012;18(23):3015-9. https://doi.org/10.3748/wjg.v18.i23.3015

33. Xu Q, Tao L, Wu Q, Gao F, Zhang F, Yuan L, He XD. Prevalences of and risk factors for biliary stones and gallbladder polyps in a large Chinese population. HPB (Oxford). 2012;14(6):373-81. https://doi.org/10.1111/j.1477-2574.2012.00457.x

34. Collett JA, Allan RB, Chisholm RJ, Wilson IR, Burt MJ, Chapman BA. Gallbladder polyps: prospective study. JUM. 1998; 17(4):207-11. https://doi.org/10.7863/jum.1998.17.4.207

35. Escalona PA, León GF, Bellolio RF, Pimentel MF, Guajardo BM, Gennero R, Cruz JP, Viviani P, Ibáñez L. Pólipos vesiculares: correlación entre hallazgos ecográficos e histopatológicos. Rev Méd Chile. 2006;134(10):1237-42. https://doi.org/10.4067/S0034-98872006001000004 